\title{
Intranasal Delivery of Morphine
}

\author{
L. ILLUM, P. WATTS, A. N. FISHER, M. HINCHCLIFFE, H. NORBURY, I. JABBAL-GILL, R. NANKERVIS, and
}

S. S. DAVIS

West Pharmaceutical Services, Drug Delivery and Clinical Research Centre Ltd., Albert Einstein Centre, Nottingham Science and Technology Park, Nottingham, United Kingdom

Received August 8, 2001; accepted December 24, $2001 \quad$ This article is available online at http://jpet.aspetjournals.org

\begin{abstract}
Morphine administered nasally to humans as a simple solution is only absorbed to a limited degree, with a bioavailability of the order of $10 \%$ compared with intravenous administration. This article describes the development of novel nasal morphine formulations based on chitosan, which, in the sheep model, provide a highly increased absorption with a 5- to 6-fold increase in bioavailability over simple morphine solutions. The chitosan-morphine nasal formulations have been tested in healthy volunteers in comparison with a slow i.v. infusion (over $30 \mathrm{~min}$ ) of morphine. The results show that the nasal formulation was rapidly absorbed with a $T_{\max }$ of 15 min or less and a bioavailability of nearly $60 \%$. The shape of the plasma profile
\end{abstract}

for nasal delivery of the chitosan-morphine formulation was similar to the one obtained for the slow i.v. administration of morphine. Furthermore, the metabolite profile obtained after the nasal administration of the chitosan-morphine nasal formulation was essentially identical to the one obtained for morphine administered by the intravenous route. The levels of both morphine-6-glucuronide and morphine-3-glucuronide were only about $25 \%$ of that found after oral administration of morphine. It is concluded that a properly designed nasal morphine formulation (such as one with chitosan) can result in a noninjectable opioid product capable of offering patients rapid and efficient pain relief.
Morphine, a potent narcotic analgesic, produces a variety of pharmacological responses by interacting with the opioid receptors in the nervous system. It is used widely for preoperative and anxiolytic therapy in pediatric patients, for the management of postoperative pain, and for moderate-to-severe pain in cancer patients because of its general availability, the choice of different formulations and routes of delivery, and the well characterized pharmacological properties. At least one-third of newly diagnosed cancer patients and about two-thirds of patients with an advanced disease experience pain either as chronic pain or as breakthrough pain episodes or both (Foley, 1995). The World Health Organization recommended in 1986 that advanced cancer pain should be treated in accordance with the analgesic ladder (World Health Organization, 1986).

Morphine is most commonly administered via the oral route, either as an oral solution, as an immediate release or controlled release oral tablet, or capsule preparation and is readily absorbed in the small intestine. Due to considerable intestinal metabolism and extensive hepatic first pass effect the oral bioavailability has been reported to be as low as $20 \%$

All authors of this article are employees of West Pharmaceutical Services and as such have an indirect interest in the outcome of the research. The employees gain no direct financial benefit from this research apart from any benefit that may arise from the impact of the results on share prices. Some of the authors have shares or share options in the company.
(Bourget et al., 1995) and 32\% (Westerling et al., 1995). The main metabolites of morphine are morphine-6-glucuronide (M-6-G), which is an active analgesic agent, and morphine3-glucuronide (M-3-G), which is inactive (Osborne et al., 1990; Westerling et al., 1995; Faura et al., 1996). Oral morphine therapy results in a range of side effects (e.g., respiratory depression, constipation, nausea, and vomiting) in the majority of patients (Twycross, 1994) and even patients with generally well controlled (chronic) pain will experience several 30-60-min periods of excruciating "breakthrough pain" every day, triggered by manipulations of the patient or appearing spontaneously (Cleary, 1997). Breakthrough pain is normally treated by oral opioid medication such as a morphine solution or oral immediate release tablets, but the maximum plasma concentration may not be reached for $0.8 \mathrm{~h}$, resulting in slow onset of analgesia.

Analgesic agents such as fentanyl, oxycodone, and butorphanol can be effectively and rapidly absorbed from the nasal cavity (due to their relative high lipophilicity) without the help of absorption promoters and thereby provide rapid onset of analgesia (Shyu et al., 1993; Takala et al., 1997). However, in humans morphine is only absorbed to a low degree when given by the nasal route and mainly when reaching the small intestine after clearance from the nasal cavity (Behl, 2000)

As shown by ourselves and other groups, the nasal absorption of small polar molecules and polypeptides can be greatly

ABBREVIATIONS: M-6-G, morphine-6-glucuronide; M-3-G, morphine-3-glucuronide; SMS, starch microspheres; LPC, lysophosphatidylcholine; HPLC, high-performance liquid chromatography; AUC, area under the plasma curve; F\%, bioavailability; ANOVA, analysis of variance. 
improved if administered in combination with an absorptionpromoting agent such as chitosan (Illum et al., 1994, 1996, 2000; Illum, 1998a; Roon et al., 1999). Hence, when M-6-G $(\log \mathrm{P}=-0.76)$, which is more hydrophilic than morphine ( $\log \mathrm{P}=0.89$ ), was formulated with a $0.5 \%$ chitosan solution the bioavailability in sheep after nasal administration was $31 \%$ relative to an intravenous injection (Illum et al., 1996).

Chitosan is a linear polysaccharide comprised of two monosaccharides: $N$-acetyl-D-glucosamine and D-glucosamine linked together by glucosidic bonds. Chitosan is produced by alkaline hydrolysis (deacetylation) of chitin obtained from crustacean shells and forms positively charged salts when dissolved in inorganic or organic acids. Chitosan is available in a wide range of molecular weights and degrees of deacetylation. The chitosan most commonly chosen for nasal delivery of drugs is the glutamate salt with a mean molecular weight of around 200 $\mathrm{kDa}$ and a degree of deacetylation of 80 to $90 \%$. Chitosan is bioadhesive and able to interact strongly with the nasal mucus layer and with the nasal epithelial cells. The clearance of chitosan formulations from the nasal cavity of sheep and humans has been shown to be significantly slower than that of simple aqueous solutions (Soane et al., 1999, 2001). Hence, nasal chitosan drug formulations provide longer time for drug transport across the nasal membrane, before the formulation is cleared by the mucociliary clearance mechanism. Furthermore, chitosan has also been shown in Caco- 2 cell culture studies to open transiently the tight junctions between cells, which enables hydrophilic drugs to pass through the membrane by the paracellular route (Dodane et al., 1999).

The purpose of the present work was to study the nasal absorption of morphine in an animal model and in humans and to develop a suitable nasal morphine formulation that could provide rapid and efficient absorption of the morphine across the nasal membrane. Various formulations, expected to enhance the nasal absorption of morphine, were tested in sheep (to include bioadhesive starch microspheres, and chitosan solution and powder formulations). Selected formulations were subsequently administered to human volunteers and the pharmacokinetic profile and tolerability of the formulations were evaluated.

\section{Experimental Procedures}

\section{Materials}

Morphine hydrochloride BP was purchased from MacFarlane Smith Ltd. (Edinburgh, Scotland, UK). Morphine sulfate $(10 \mathrm{mg} / \mathrm{ml})$ in a sterile saline solution was obtained from Martindale Pharmaceuticals (Essex, UK). Chitosan glutamate (Sea Cure G + 210) and chitosan hydrochloride (Sea Cure Cl 113) were obtained from Pronova (Drammen, Norway). The chitosan was supplied spray dried and had the form of microspheres. Crosslinked Eldexomer starch microspheres (SMS) were supplied by Perstorp Pharma (Perstorp, Sweden) and the L- $\alpha$-lysophosphatidylcholine (LPC) by Sigma-Aldrich (Poole, Dorset, UK). All other materials were of pharmaceutical grade or at least analytical grade.

Prototype devices from Bespak Ltd. (King's Lynn, UK) were used to administer the powder formulations to the nasal cavity in the human clinical trial. These single dose devices contained a polypropylene capsule loaded with the correct dose of powder formulation. The capsule was pierced by priming the device and the dose delivered by the volunteer breathing in rapidly through one nostril.

The dosing devices used in the clinical trial for administration of a single dose of a liquid formulation were supplied by Pfeiffer $\mathrm{GmbH}$
(Radolfzell, Germany). The dose was contained in a glass vial, which was assembled into the dosing unit. Each device was calibrated to deliver a dose of $125 \mu \mathrm{l}$, for which a loading of $145 \mu \mathrm{l}$ was required. The dose was released as a spray into the nasal cavity by pressing the plunger on the device.

\section{Formulation Preparation}

Formulations Used in Sheep Studies. A summary of the morphine formulations administered to the sheep is given in Table 1. The morphine solution for intravenous injection (formulation 1) was prepared by dissolving $40 \mathrm{mg}$ of morphine hydrochloride in $50 \mathrm{ml}$ of sterile isotonic saline and filtering through a sterile $(0.2-\mu \mathrm{m}) \mathrm{mem}-$ brane filter (Satorius, Gottingen, Germany). The osmolality of this solution was $0.292 \mathrm{Osmol} / \mathrm{kg}$.

The nasal morphine control solution (formulation 2) was prepared by dissolving $150 \mathrm{mg}$ of morphine hydrochloride in $4 \mathrm{ml}$ of $0.5 \%$ sodium chloride solution ( $\mathrm{pH}$ adjusted to 4 with $1 \mathrm{M} \mathrm{HCl}$ ) and making up the volume to $5 \mathrm{ml}$ with the $0.5 \%$ sodium chloride solution. The final morphine solution had a morphine hydrochloride concentration of $30 \mathrm{mg} / \mathrm{ml}$ and a $\mathrm{pH}$ of 4.02 .

The nasal morphine chitosan solution formulation (formulation 3) was prepared by dissolving $50 \mathrm{mg}$ of chitosan glutamate in $10 \mathrm{ml}$ of $0.5 \%$ sodium chloride solution ( $\mathrm{pH}$ adjusted to 4 with $1 \mathrm{M} \mathrm{HCl})$ and filtering through a $0.2-\mu \mathrm{m}$ membrane filter (Satorius). Morphine hydrochloride $(150 \mathrm{mg}$ ) was added to $5 \mathrm{ml}$ of this chitosan solution. The final formulation contained $30 \mathrm{mg} / \mathrm{ml}$ morphine hydrochloride in an isotonic (osmolality of $0.301 \mathrm{Osmol} / \mathrm{kg}$ ) $0.5 \%$ chitosan glutamate solution at $\mathrm{pH} 3.81$.

The nasal morphine chitosan microsphere formulation (formulation 4) was prepared by suspending $800 \mathrm{mg}$ of cross-linked chitosan microspheres (prepared using a conventional water-in-oil emulsification technique) in $10 \mathrm{ml}$ of distilled water and adding $5 \mathrm{ml}$ of a 24.0 $\mathrm{mg} / \mathrm{ml}$ morphine hydrochloride solution and $38 \mathrm{ml}$ of distilled water. The mixture was stirred for $20 \mathrm{~min}$ and freeze-dried by using an Edwards Modulyo $4 \mathrm{~K}$ freeze-dryer (Edwards High Vacuum Int., Crawley, UK). The powder was stored desiccated at $4^{\circ} \mathrm{C}$ until use.

The nasal formulation of morphine based on starch microspheres (formulation 5) was prepared by suspending $800 \mathrm{mg}$ of SMS in $10 \mathrm{ml}$ of distilled water. Five milliliters of a $24.0 \mathrm{mg} / \mathrm{ml}$ morphine hydrochloride solution and $10 \mathrm{ml}$ of an $8 \mathrm{mg} / \mathrm{ml} \mathrm{LPC}$ solution were added together with $28 \mathrm{ml}$ of distilled water. The mixture was stirred for 20 min then frozen using liquid nitrogen and freeze-dried using an Edwards Modulyo 4K freeze-dryer (Edwards High Vacuum Int.). The powder was stored desiccated at $4^{\circ} \mathrm{C}$ until use.

Formulations Used in Human Studies. A summary of the morphine formulations administered to human volunteers is given in Table 2.

The nasal morphine formulation based on chitosan powder (formulation A) was prepared by blending manually $1.3 \mathrm{~g}$ of morphine hydrochloride and $6.7 \mathrm{~g}$ of chitosan glutamate by using a mortar and pestle. After ensuring that the powder blend was homogenous according to specifications ( $16.5 \pm 1.65 \%$ morphine hydrochloride) $30.3 \pm 0.3 \mathrm{mg}$-portions (equivalent to $5 \mathrm{mg}$ of morphine hydrochloride) were weighed into the polypropylene capsules for use in the Bespak devices.

The nasal solution formulation of morphine containing chitosan (formulation B) $(50 \mathrm{ml})$ was prepared by dissolving $0.25 \mathrm{~g}$ of chitosan glutamate in ultrapure water, adding $2.0 \mathrm{~g}$ of morphine hydrochloride and $0.185 \mathrm{~g}$ of sodium chloride and adjusting the $\mathrm{pH}$ to 4 with 1 $\mathrm{M}$ hydrochloric acid. The solution was passed through a $0.2-\mu \mathrm{m}$ membrane filter (Sartorius) and divided into the Pfeiffer nasal spray devices in aliquots of $145 \mu \mathrm{l}$. Each device would deliver $125 \mu \mathrm{l}$ of formulation equivalent to $5 \mathrm{mg}$ of morphine hydrochloride.

Formulation C was a commercial (Martindale Pharmaceuticals) $10 \mathrm{mg} / \mathrm{ml}$ morphine sulfate injection formulation supplied in 1.1-ml ampoules.

The microbial content of the human nasal formulations was tested by a commercial laboratory (International Laboratory Services Ltd., 
TABLE 1

Summary of compositions of morphine formulations administered to sheep Doses are given on the basis of per kilogram sheep.

\begin{tabular}{|c|c|c|c|c|c|c|}
\hline Formulation & $\begin{array}{l}\text { Morphine } \\
\mathrm{HCl}\end{array}$ & $\begin{array}{l}\text { Chitosan } \\
\text { Glutamate }\end{array}$ & $\begin{array}{c}\text { Chitosan } \\
\text { (XL-CHI MS) }\end{array}$ & SMS & $\mathrm{LPC}$ & $\begin{array}{l}\text { Volume/kg or } \\
\text { Weight/kg } \\
\text { Administered }\end{array}$ \\
\hline & & & $m g$ & & & \\
\hline 1. i.v. Sol & 0.1 & & & & & $0.125 \mathrm{ml}$ \\
\hline 2. IN Sol & 0.3 & & & & & $0.01 \mathrm{ml}$ \\
\hline 3. IN Sol + CHI & 0.3 & 0.05 & & & & $0.01 \mathrm{ml}$ \\
\hline 4. IN PWD XL-CHI & 0.3 & & 2.0 & & & $2.3 \mathrm{mg}$ \\
\hline 5. IN PWD SMS + LPC & 0.3 & & & 2.0 & 0.2 & $2.5 \mathrm{mg}$ \\
\hline
\end{tabular}

CHI, chitosan glutamate; IN, intranasal; PWD, powder; Sol, solution; XL-CHI, cross-linked chitosan microspheres.

TABLE 2

Summary of compositions of morphine formulations administered to human volunteers

\begin{tabular}{cccc}
\hline Formulation & Morphine & Chitosan Glutamate & Dose/Volunteer \\
\hline A. IN PWD CHI & $16.5 \%(\mathrm{w} / \mathrm{w})^{a}$ & $83.4 \%(\mathrm{w} / \mathrm{w})$ & $10 \mathrm{mg}$ of morphine \\
$(60.6-\mathrm{mg}$ dose $)$ & $42.7 \mathrm{mg} / \mathrm{ml}^{a}$ & $5.0 \mathrm{mg} / \mathrm{ml}$ & $50.6 \mathrm{mg}$ of CHI \\
B. IN Sol CHI & $10 \mathrm{mg} / \mathrm{ml}^{b}$ & & $10 \mathrm{mg}$ of morphine \\
$(250-\mu$ l dose $)$ & & $1.25 \mathrm{mg}$ of CHI \\
C. i.v. Sol & & $10 \mathrm{mg} \mathrm{of} \mathrm{morphine}^{b}$ \\
$(1.0-\mathrm{ml}$ dose $)$ & & \\
\hline
\end{tabular}

CHI, chitosan glutamate; IN, intranasal; PWD, powder; Sol, solution.

${ }^{a}$ Morphine hydrochloride.

${ }^{b}$ Morphine sulfate.

Shardlow, UK) and was found to be within United States Pharmacopeia specifications with a total viable count of $<5$ colony-forming units/g before the start of the trial and after the trial.

\section{Analytical Methods}

In Vitro Morphine Assay. The morphine hydrochloride analysis was performed by reverse phase HPLC with ultraviolet detection by using a method slightly modified from the assay method described by Svensson et al. (1982). The limit of detection of the assay was 10 $\mu \mathrm{g} / \mathrm{ml}$.

For the liquid formulations, the samples were diluted 1000 times in HPLC mobile phase and analyzed in duplicate. For the powder formulations the 30-mg samples were dispersed in $26 \mathrm{ml}$ of acetonitrile and made up to $100 \mathrm{ml}$ with HPLC buffer. Each sample was filtered through $0.8-\mu \mathrm{m}$ filters (Sartorius) and analyzed in duplicate by HPLC. The calibration curve used for all samples covered the concentration range 10 to $100 \mu \mathrm{g} / \mathrm{ml}$.

Plasma Morphine Levels in Sheep. The plasma morphine levels were measured in the sheep plasma samples by a solid phase quantitative radioimmunoassay, by using a commercial Coat-ACount serum morphine kit (Diagnostics Product Corporation, Abingdon, Oxfordshire, UK). The RIA-CALC program was used for calculating the plasma morphine concentrations in nanomoles per milliliter, by using a morphine calibration curve. All measurements were performed using plasma samples. Validation of the assay showed the intraday and interday variation to be within the acceptable range. The coefficient of variation was less than $15 \%$ for all the quality control samples (low, medium, and high). The limit of detection was found to be $2.8 \mathrm{nM}$. The cross-reactivity of the method with M-6-G and M-3-G metabolites was reported as negligible. All samples were analyzed at least in duplicate.

The curves for the intravenous dosing were extrapolated to zero by using the Minim program (Minin 2.0.3; R. D. Purves, University of Utago, Utago, New Zealand) and were used to calculate the area under the plasma curve (AUC) values. The AUC values for the nasally dosed animals were calculated using the Excel program. Values for the time to peak plasma concentration $\left(T_{\max }\right)$, peak concentration $\left(C_{\max }\right)$, AUC, and bioavailability $(\mathrm{F} \%)$ were calculated.

Plasma Morphine Levels in Human Volunteers. The plasma samples were analyzed by HPLC for morphine, morphine-6-glucuro- nide, and morphine-3-glucuronide by Hafslund Nycomed Pharma (Linz, Austria). The extraction method used was a modification of the method described by Murphey et al. (1993) and the HPLC conditions used based on the method described by Todd et al. (1982). The method was shown to be linear over the chosen concentration ranges and stability of the analytes was demonstrated in the injection solution. A series of quality control samples were included in each extraction and accuracy and precision were demonstrated to deviate by less than $20 \%$ for morphine. Pharmacokinetic analysis was performed using the program TOPFIT version 2.0 according to noncompartmental methods.

\section{Sheep Studies}

The sheep nasal model was chosen for the initial studies because it has been shown in various studies and by various groups that this model is very predictive of results in humans (Illum, 1996). Twenty male, cross-bred Texel and Suffolk sheep of $49.1 \pm 12.1 \mathrm{~kg}$ (mean \pm S.D.) were used in the study and divided into five groups of four animals. The sheep were housed indoors for the duration of the study and fed ad libitum on a nut concentrate and hay. The animals were not fasted before the experiment. On the first day of the study, an indwelling Secalon cannula fitted with a flow switch was placed approximately $15 \mathrm{~cm}$ into one of the external jugular veins of each animal. The cannulae were kept patent by flushing with heparinized (25 IU/ml) $0.9 \%$ saline solution. On the second day, the sheep were sedated for about $3 \mathrm{~min}$ with an intravenous dose of $100 \mathrm{mg} / \mathrm{ml}$ ketamine (Vetalar; Fort Dodge Animal Health, Ltd., Southhampton, UK) at $2.25 \mathrm{mg} / \mathrm{kg}$ during dosing to prevent sneezing. The solution formulations were instilled nasally from a 1-ml syringe $(0.01 \mathrm{ml} / \mathrm{kg})$ attached to a blueline umbilical cannula inserted approximately 8 $\mathrm{cm}$ into the nasal cavity. The dose was divided equally between the two nostrils. The powder formulations were administered nasally using a blueline siliconized oral/nasal tracheal tube containing the preweighed dose, inserted approximately $8 \mathrm{~cm}$ into the nasal cavity, by means of a simple one-way spray bellows. The intravenous administration was given as a slow injection $(0.125 \mathrm{ml} / \mathrm{kg}$ over $1 \mathrm{~min})$ via the indwelling jugular vein cannula. The cannula was flushed with $10 \mathrm{ml}$ of sterile normal saline. Blood samples of $4.0 \mathrm{ml}$ were collected from the cannulated jugular vein of the sheep at 20,15 , and 5 min before morphine administration and at 5, 10, 15, 20, 30, 45, 60, 
$90,120,150,180,240,300$, and $360 \mathrm{~min}$ after dosing. For the intravenous administration an extra blood sample was collected at 2 min after administration. The blood samples were gently mixed in 4 $\mathrm{ml}$ of heparinized tubes (60 IU of lithium heparin; Sarstedt, Leicester, UK) and kept on crushed ice until plasma separation. The plasma samples were stored at $-20^{\circ} \mathrm{C}$ awaiting analysis. The cannulae were removed upon completion of the study and the sheep returned to their normal housing. The animal studies were performed under an approved Home Office Animal Project License in accordance with the Animals (Scientific Procedures) Act 1986.

\section{Human Volunteer Trial}

The study was conducted as a three-way crossover design in 12 healthy volunteers (male and female) between 18 and 36 years of age. The volunteers were fasted from 10:00 PM the night before each dose administration. A light breakfast was allowed $2 \mathrm{~h}$ postdose. Lunch and evening meals were provided at 5 and $10 \mathrm{~h}$ postdose, respectively. A cannula was inserted into a vein in the lower arm for blood sampling at the start of each study day. The volunteers received the three morphine formulations (A, B, or C) in a randomized order according to a Latin square design. There was a 1-week washout period between the administration of the various doses. Before recruitment into the trial, volunteers were given detailed information about the study and signed a consent form. They then underwent a medical screening procedure, including a physical examination, medical history, clinical laboratory tests, and ECG recording, according to the protocol. Only volunteers complying with the inclusion and exclusion criteria were used in the study. No volunteer with a history of intravenous drug abuse or abuse of opioids was included in the study. The clinical protocol was approved by an Ethics Committee and the study carried out at Medeval Ltd. (Skelton House, Manchester Science Park, Manchester, UK) in accordance with the Declaration of Helsinki.

The nasal solution and powder formulations were administered by a trained nurse or clinician to the volunteers according to written instructions. The volunteers received the content of a capsule in each nostril (a nominal $10 \mathrm{mg}$ of morphine hydrochloride) for the powder formulation and $125 \mu \mathrm{l}$ in each nostril (10 mg of morphine hydrochloride) for the solution formulation. Ten milligrams of morphine sulfate was infused over a period of $30 \mathrm{~min}$ via an indwelling intravenous catheter in a forearm vein that was not used for blood sampling. The infusions were prepared by adding $18 \mathrm{ml}$ of sterile normal saline to $2 \mathrm{ml}$ of the morphine sulfate commercial preparation. After priming the giving set, the infusion pumps were set to infuse $20 \mathrm{ml} / \mathrm{h}$ for $30 \mathrm{~min}$ giving a total of $10 \mathrm{mg}$ of morphine sulfate.

The formulations to be tested in the human studies were selected from the sheep studies on the basis of an evaluation of potential toxicological problems that might be encountered in the clinic for some of the formulations.

The residual doses left in the nasal devices were analyzed by HPLC for morphine content and the exact doses delivered to each volunteer calculated by subtracting the residual morphine dose from the original dose in the device. All pharmacokinetic results were adjusted to account for the dose given. The mean residual doses constituted less than $20 \%$ of the total dose.

Blood samples $(8 \mathrm{ml}$ ) were taken at $-15 \mathrm{~min}$ (before dosing) and at $5 \mathrm{~min}, 15 \mathrm{~min}, 30 \mathrm{~min}, 32 \mathrm{~min}, 35 \mathrm{~min}, 40 \mathrm{~min}, 45 \mathrm{~min}, 1 \mathrm{~h}, 1 \mathrm{~h} 15$ $\min , 1 \mathrm{~h} 30 \mathrm{~min}, 2 \mathrm{~h}, 3 \mathrm{~h}, 4 \mathrm{~h}, 6 \mathrm{~h}, 8 \mathrm{~h}$, and $12 \mathrm{~h}$ postdose for the intravenous administration of morphine or $5 \mathrm{~min}, 10 \mathrm{~min}, 15 \mathrm{~min}, 30$ min, $45 \mathrm{~min}, 1 \mathrm{~h}, 1 \mathrm{~h} 30 \mathrm{~min}, 2 \mathrm{~h}, 2 \mathrm{~h} 30 \mathrm{~min}, 3 \mathrm{~h}, 4 \mathrm{~h}, 6 \mathrm{~h}, 8 \mathrm{~h}$, and $12 \mathrm{~h}$ postdose for the nasal doses. The total volume of blood sampled during the whole study was approximately $490 \mathrm{ml}$ from each volunteer. The samples were collected into heparinized tubes and maintained on ice until centrifugation. The samples were centrifuged within $15 \mathrm{~min}$ of collection on a refrigerated centrifuge at $4^{\circ} \mathrm{C}$ at $2000 \mathrm{~g}$ for $10 \mathrm{~min}$. The resultant plasma was divided into two samples of 2.5 and $1.5 \mathrm{ml}$ and stored at $-20^{\circ} \mathrm{C}$ until analysis.

At specified times after dosing the volunteers were asked to complete a form describing the taste and tolerability of the drug formulation in the nasal cavity on a scale from 0 to 10. A questionnaire was used to record the central effects of the morphine such as the degree of drowsiness and nausea on a similar scale from 0 to 10 . For each time point, the total score for all volunteers and the number of volunteers recording a score greater than zero are recorded. The maximum score for each type of intolerability or central effect is 120 and the maximum total intolerability score is 600 .

Blood pressure, respiratory rate, and heart rate were monitored before dosing and at specific times afterward. Volunteers were closely monitored for effects on the central nervous system for the duration of the study, especially in the first $2 \mathrm{~h}$ after dose administration.

\section{Statistical Analysis}

Statistical analysis of data obtained from the sheep/human studies was performed using GraphPad Instat software (GraphPad Software, San Diego, CA). Throughout, the level of statistical significance was chosen as $p<0.05$. For comparison of intravenous and/or nasal sheep data, a one-way analysis of variance (ANOVA) with Tukey-Kramer multiple comparisons post test was used. The post test was performed only if findings of the ANOVA were significant. Analysis of human nasal and intravenous data was by one-way ANOVA with Tukey-Kramer multiple comparison post test as appropriate. Comparison of data from the two nasal groups was performed using unpaired (two-tailed) $t$ tests.

TABLE 3

Pharmacokinetic parameters ( \pm S.D.) for morphine administered nasally in sheep $(n=4)$

Only significant relations are indicated, for all other comparisons made $p>0.05$.

\begin{tabular}{lcccc}
\hline \multicolumn{1}{c}{ Formulation } & $T_{\max }$ & $C_{\max }$ & AUC & F \\
& $\min$ & $n \operatorname{mol} / l$ & $n m o l / l \cdot m i n$ & $\%$ of i.v. \\
i.v. Sol & $2.0 \pm 0.0^{a, b}$ & $2592.6 \pm 3589.5$ & $21505 \pm 21615$ & 100 \\
IN Sol & $20.0 \pm 7.1^{a, c, d, f, g}$ & $151.2 \pm 64.3^{h}$ & $6799 \pm 2143^{e, i}$ & $10.5 \pm 3.3^{k}$ \\
IN Sol + CHI & $13.8 \pm 2.5^{b}$ & $657.0 \pm 491.0$ & $17169 \pm 9376^{j}$ & $26.6 \pm 14.5^{l}$ \\
IN PWD XL-CHI & $7.5 \pm 2.9^{c, f}$ & $1010.8 \pm 733.4$ & $35197 \pm 18606$ & $54.6 \pm 28.8$ \\
IN PWD SMS + LPC & $10.0 \pm 4.1^{d, g}$ & $1875.9 \pm 1125.3^{h}$ & $48235 \pm 18825^{e, i, j}$ & $74.8 \pm 29.2^{k, l}$ \\
One-way ANOVA & $p<0.001$ & $p>0.05$ & $p<0.05$ & NA \\
One-way ANOVA** & $p<0.05$ & $p<0.05$ & $p<0.01$ & $p<0.01$ \\
\hline
\end{tabular}

CHI, chitosan glutamate; IN, intranasal; PWD, powder; Sol, solution; XL-CHI, cross-linked chitosan microspheres.

${ }_{a} p<0.001$, comparisons made of all formulations (1-5).

${ }_{b, c} p<0.01$, comparisons made of all formulations (1-5).

$d, e p<0.05$, comparisons made of all formulations (1-5).

${ }_{f, i, k} p<0.05$, comparisons made of all formulations $(1-5)$.
$g, h, j, l$

$g, h_{3}, l p<0.05$, comparisons made only of the nasal formulations (2-5)

* Tukey-Kramer multiple comparisons test following ANOVA, comparisons made of all formulations (1-5),

** Tukey-Kramer multiple comparisons test following ANOVA, comparisons made only of the nasal formulations (2-5). 


\section{Results}

Sheep Studies. The pharmacokinetic values for the nasal absorption of morphine in sheep are shown in Table 3 and the plasma profiles for the nasal formulations for the first 120 min after dosing are given in Fig. 1. The absorption of morphine across the nasal membrane from a nasal morphine hydrochloride solution formulation given as a control (formulation 2) was limited with a $C_{\max }$ of $151 \mathrm{nM}$ and an $\mathrm{F} \%$ in the order of $10 \%$. The $T_{\max }$ of $20 \mathrm{~min}$ indicated relatively slow rate of nasal absorption of morphine from the control formulation. When $0.5 \%$ chitosan was coadministered with morphine in a solution formulation (formulation 3) the nasal absorption was increased with a $C_{\max }$ of $657 \mathrm{nM}$ and a bioavailability of $26.6 \%$. The rate of absorption was also improved with $T_{\max }$ at about $14 \mathrm{~min}$. Chitosan formulated into microspheres and administered with the morphine (formulation 4) further improved nasal morphine absorption. The $C_{\max }$ was found to be $1010 \mathrm{nM}$, the $T_{\max }$ about $8 \mathrm{~min}$, and the bioavailability $54.6 \%$, representing more than a 4 -fold increase in absorption compared with the morphine control solution formulation. Still further improvement in nasal morphine absorption was observed after dosing a powder formulation comprising starch microspheres, LPC, and morphine (formulation 5); values of $C_{\max }, T_{\max }$, and $\mathrm{F} \%$ of $1875 \mathrm{nM}, 10 \mathrm{~min}$, and 75\%, respectively, were recorded.

Statistical comparison of the nasal dose groups showed that the starch microspheres/LPC formulation significantly improved $(p<0.05)$ the nasal $\mathrm{F} \%$ of morphine compared with the morphine control and chitosan-based solution formulations, although differences between the chitosan-based formulations were not significant $(p>0.05)$. After dosing the chitosan- and starch-based microsphere formulations to sheep, values of $T_{\max }$ were significantly lower $(p<0.05)$ than those obtained after dosing the nasal control formulation, indicating faster absorption of morphine from the powder

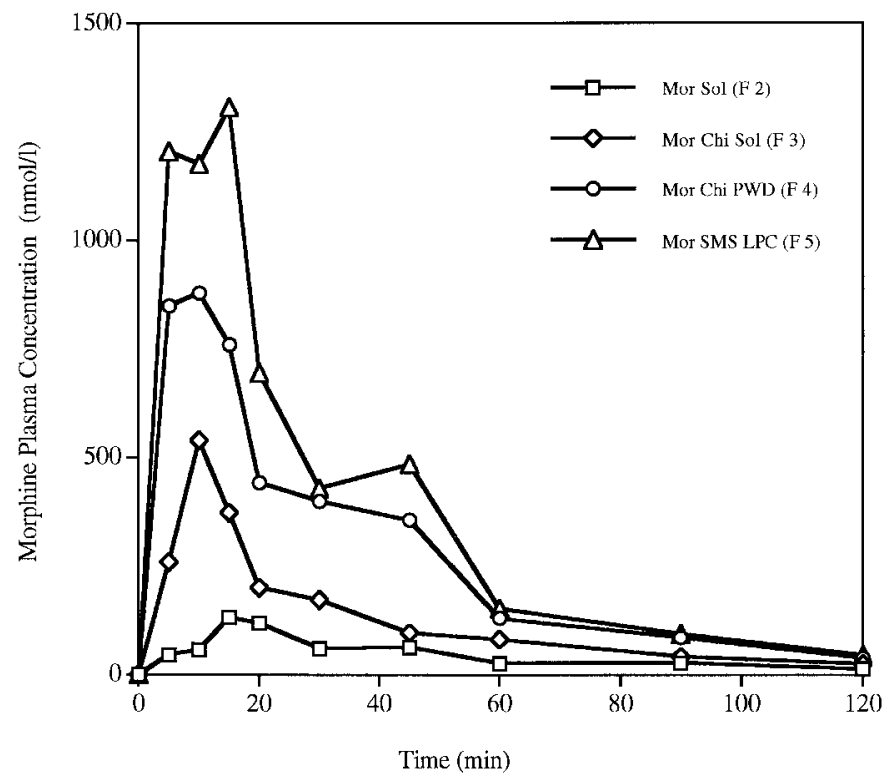

Fig. 1. Morphine plasma concentration after nasal administration of morphine formulations in sheep. Mor Sol, morphine solution; Mor Chi Sol, morphine solution containing chitosan; Mor Chi PWD, morphinechitosan powder; Mor SMS LPC, starch microspheres with lysophosphatidylcholine and morphine as a freeze-dried powder. formulations. Further details of all statistical comparisons can be found in Table 3.

Human Phase I Clinical Trial. The pharmacokinetic values for the nasal absorption of morphine in human volunteers are shown in Table 4 and the plasma profiles for the nasal and intravenous formulations are given in Fig. 2. After slow intravenous administration of $10 \mathrm{mg}$ of morphine sulfate the mean plasma concentration of morphine $\left(C_{\max }\right)$ was $336 \pm 68 \mathrm{nM}, 30 \mathrm{~min}$ after the start of dose administration. The plasma half-life of morphine was $1.67 \pm 0.26 \mathrm{~h}$. After nasal administration of a solution formulation containing $0.5 \%$ chitosan and morphine hydrochloride (formulation B, nominal dose $10 \mathrm{mg}$ of morphine per volunteer) peak plasma concentrations of morphine were rapidly attained $\left(C_{\max }\right.$ of $98 \pm 57 \mathrm{nM}, T_{\max }$ of $16 \pm 7 \mathrm{~min}$ ). The shape of the plasma morphine profile was similar to that obtained after the slow intravenous injection (Fig. 2). The plasma half-life $\left(t_{1 / 2}\right)$ obtained after the nasal solution formulation $(2.98 \pm 2.39 \mathrm{~h})$ was not significantly different $(p>0.05)$ from that after slow intravenous morphine administration. The mean bioavailability of the nasal chitosan-morphine formulation was $56 \pm$ $27 \%$. For the nasal powder formulation comprising chitosan and morphine hydrochloride (formulation A, nominal dose 10 $\mathrm{mg}$ of morphine per volunteer) the results were not significantly different $(p>0.05)$ from those of the chitosan-based solution formulation and the shape of the plasma morphine profile obtained was similar. Values of $C_{\max }, T_{\max }, t_{1 / 2}$, and F\% were $92 \pm 36 \mathrm{nM}, 21 \pm 7 \mathrm{~min}, 2.72 \pm 2.17 \mathrm{~h}$, and $56 \pm$ $20 \%$, respectively.

The lack of statistically significant differences between the pharmacokinetic parameters could be attributed to the relatively small sample size (12 subjects) in the study. Based on the values of S.D. obtained for the parameter F\% in human subjects and assuming that a difference of $\pm 10 \%$ between mean values of $\mathrm{F} \%$ would be the smallest difference of scientific interest, it is estimated that groups of around 80 subjects would be required to demonstrate differences (at 70\% statistical power) between the chitosan powder and chitosan solution formulations (GraphPad StatMate software).

The results from the questionnaire on tolerability after administration of the three morphine formulations are summarized in Table 5. It can be seen that for the two nasal formulations the summarized total scores (summary of total scores at each time point for all volunteers) were $30 \%$ or less of the possible maximum tolerance score of 600 . Both nasal formulations were generally well tolerated by the volunteers. However, although statistical analysis (ANOVA followed by $t$ tests) showed no significant difference $(p>0.05$ for all groups) between mean scores for each time point and symptom, the summarized total scores indicated that the solution formulation was better tolerated than the powder formulation. The effects of the powder formulation were immediate with soreness, stuffiness, and runny nose being reported within the first $5 \mathrm{~min}$ and lasting for 15 to $30 \mathrm{~min}$. For the nasal solution formulations the main effects reported were nasal stuffiness, as well as transient taste disturbance, runny nose, and soreness of the nose.

The results from the questionnaire on central effects of the morphine showed that these were experienced from all three morphine formulations. Figure 3 shows the total score for all volunteers at each time point for sedation and nausea for the three formulations. The most marked effect was sedation, 
TABLE 4

Pharmacokinetic parameters ( \pm S.D.) for morphine administered by the intravenous route and nasally in human volunteers $(n=12)$ Only significant relations are indicated, for all other comparisons made $p>0.05$.

\begin{tabular}{|c|c|c|c|c|c|}
\hline Formulation & $T_{\max }$ & $C_{\max }$ & AUC & $t_{1 / 2}$ & $\mathrm{~F}$ \\
\hline & $\min$ & $\mathrm{nmol} / \mathrm{l}$ & $\mathrm{nmol} / \mathrm{l} \cdot \mathrm{h}$ & $h$ & \% of i.v. \\
\hline IN PWD CHI & $21 \pm 7^{a}$ & $92.47 \pm 35.8^{c}$ & $176.30 \pm 64.4^{e}$ & $2.72 \pm 2.1$ & $56.0 \pm 20.0$ \\
\hline IN Sol CHI & $16 \pm 7^{b}$ & $97.72 \pm 57.2^{d}$ & $180.03 \pm 94.6^{f}$ & $2.98 \pm 2.3$ & $56.0 \pm 27.0$ \\
\hline IV Sol & $30 \pm 0^{a, b}$ & $336.36 \pm 67.6^{c, d}$ & $310.27 \pm 69.4^{e, f}$ & $1.67 \pm 0.26$ & \\
\hline One-way ANOVA* & $p<0.001$ & $p>0.001$ & $p<0.001$ & $p>0.05$ & N.A. \\
\hline$t$ test (two-tailed) $* *$ & $p>0.05$ & $p>0.05$ & $p>0.05$ & $p>0.05$ & $p>0.05$ \\
\hline
\end{tabular}

CHI, chitosan glutamate; IN, intranasal; PWD, powder; Sol, solution; N.A., not applicable.

${ }^{a} p<0.01$, comparisons made of all formulations (A-C).

$b, c, d, e, f p<0.001$, comparisons made of all formulations (A-C).

* Tukey-Kramer multiple comparisons test following ANOVA, comparisons made of all formulations (A-C).

** Tukey-Kramer multiple comparisons test following ANOVA, comparisons made only of the nasal formulations (A-B).

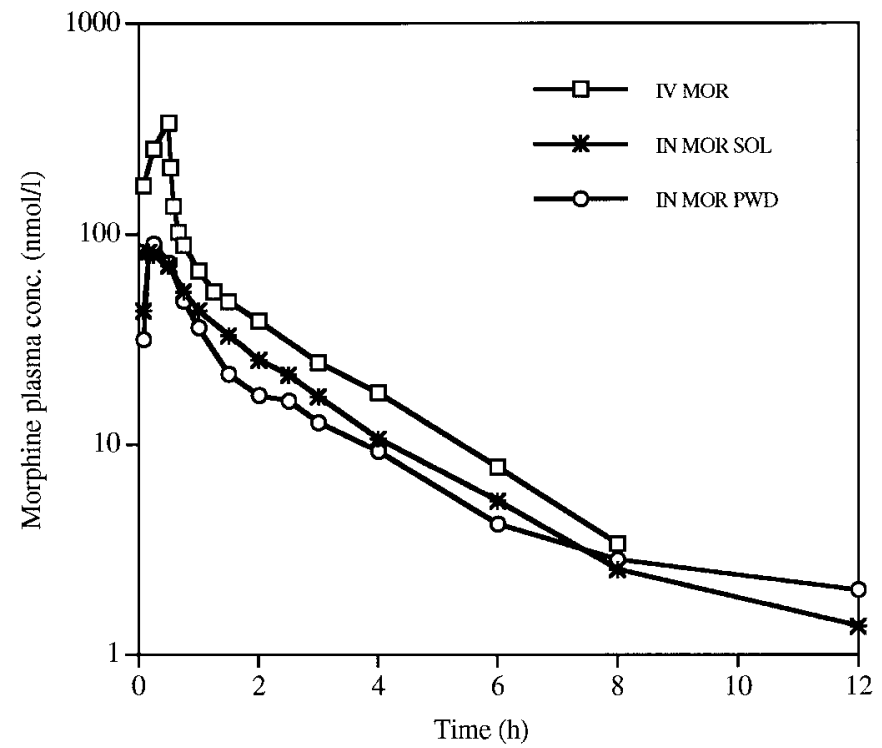

Fig. 2. Morphine plasma concentration in human volunteers after intravenous administration of morphine and after nasal administration of morphine as chitosan solution and powder formulations. Mor Chi Sol, morphine solution containing chitosan; Mor Chi PWD, morphine-chitosan powder; IN, intranasal.

which rapidly increased with time for the intravenous morphine to reach a plateau effect at $30 \mathrm{~min}$. The sedation effects from the nasal formulations increased more steadily with time within the 60 -min observation period. Although there was no significant difference ( $p>0.05$ for all groups) between the mean scores for all volunteers for sedation (data not shown), the total scores in Fig. 3 indicated that after 15 min sedation was more pronounced with intravenous than with the nasal formulations. In terms of nausea the total score showed no apparent difference between the formulations, which was supported by nonsignificance between the mean scores $(p>0.05$ for all time points). The total scores for nausea were generally very low, indicating that this was a minor effect.

The plasma profiles for the main morphine metabolites M-3-G and M-6-G are shown in Fig. 4, A and B. Figure 4A shows the metabolite profiles after the intravenous infusion of morphine and Fig. 4B after nasal administration of the chitosan-morphine solution formulation. The profile for the nasal powder formulation was not significantly different to the one for the solution formulation and hence the data are not included. The major metabolite after both i.v. and nasal administration of morphine was M-3-G with $C_{\max }$ of 415 and
$212 \mathrm{nM}$, respectively, for i.v. and nasal administration. The corresponding values for the M-6-G metabolite were $C_{\max }$ of 68 and $41 \mathrm{nM}$, respectively. The ratios between $\mathrm{AUC}_{\mathrm{M}-3-\mathrm{G}}$ and $\mathrm{AUC}_{\mathrm{M}-6-\mathrm{G}}$ were similar with values of 3.8 and 3.4 , respectively, for the intravenous dosing and administration of the nasal morphine solution. Figure 5 shows the relative levels of morphine metabolites produced after intravenous, nasal, and oral administration of morphine. The data for the oral morphine metabolites have been taken from the studies of Osborne et al. (1990). It can be seen that the relative levels of M-3-G and M-6-G metabolites are very similar for the intravenous and the nasal routes of administration and are about $25 \%$ of the levels produced after oral administration of morphine during the time period of the study.

\section{Discussion}

In the management of breakthrough pain it is of importance to use a drug and a route of administration that will provide a time-action profile characterized by rapid onset and early peak effect and duration commensurate with the span of most breakthrough pain situations. Hence, a pure $\mu$-opioid agonist such as morphine, with relatively short plasma halflife, administered nasally with an adequate delivery system would be a suitable choice.

Very few studies on the nasal delivery of morphine to humans have been published. Chast et al. (1992) administered $20 \mathrm{mg}$ of morphine acetate to six postoperative patients by the nasal and oral routes and reported a peak plasma concentration $15 \mathrm{~min}$ after nasal and $30 \mathrm{~min}$ after oral administration. The plasma profile after nasal administration was very similar to that seen after parenteral administration. However, in the article, the bioavailability was not disclosed, although pharmacokinetic data were given. Recently, data on the nasal delivery of morphine sulfate to humans as a simple solution were presented by Behl (2000). The nasal administration of morphine provided a plasma profile very similar to that found after oral administration, most likely due to an expected limited nasal absorption of the hydrophilic drug followed by a more extensive oral absorption after clearance from the nasal cavity in humans.

Studies in Sheep. Because of its polar nature, morphine is not easily transported across the nasal membrane with a bioavailability of only $10.5 \%$ in the sheep model (Fig. 1; Table 3 ). Due to the special nature of the sheep stomach (rumen) the absorption profile obtained in the sheep model can be credited to purely nasal absorption. The absorption found herein is much lower than that reported by Kondo et al. 
TABLE 5

Total nasal tolerance scores and mean scores $( \pm \mathrm{SD})$ in human volunteers after administration of a solution or a powder morphine-chitosan formulation

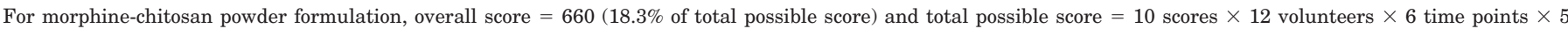

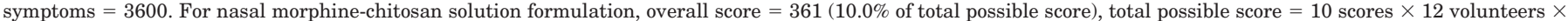
6 time points $\times 5$ symptoms $=3600$, total nasal score $=$ total score for all 12 volunteers $(0$ to 120$)$, and mean score $=$ total score divided by 12 .

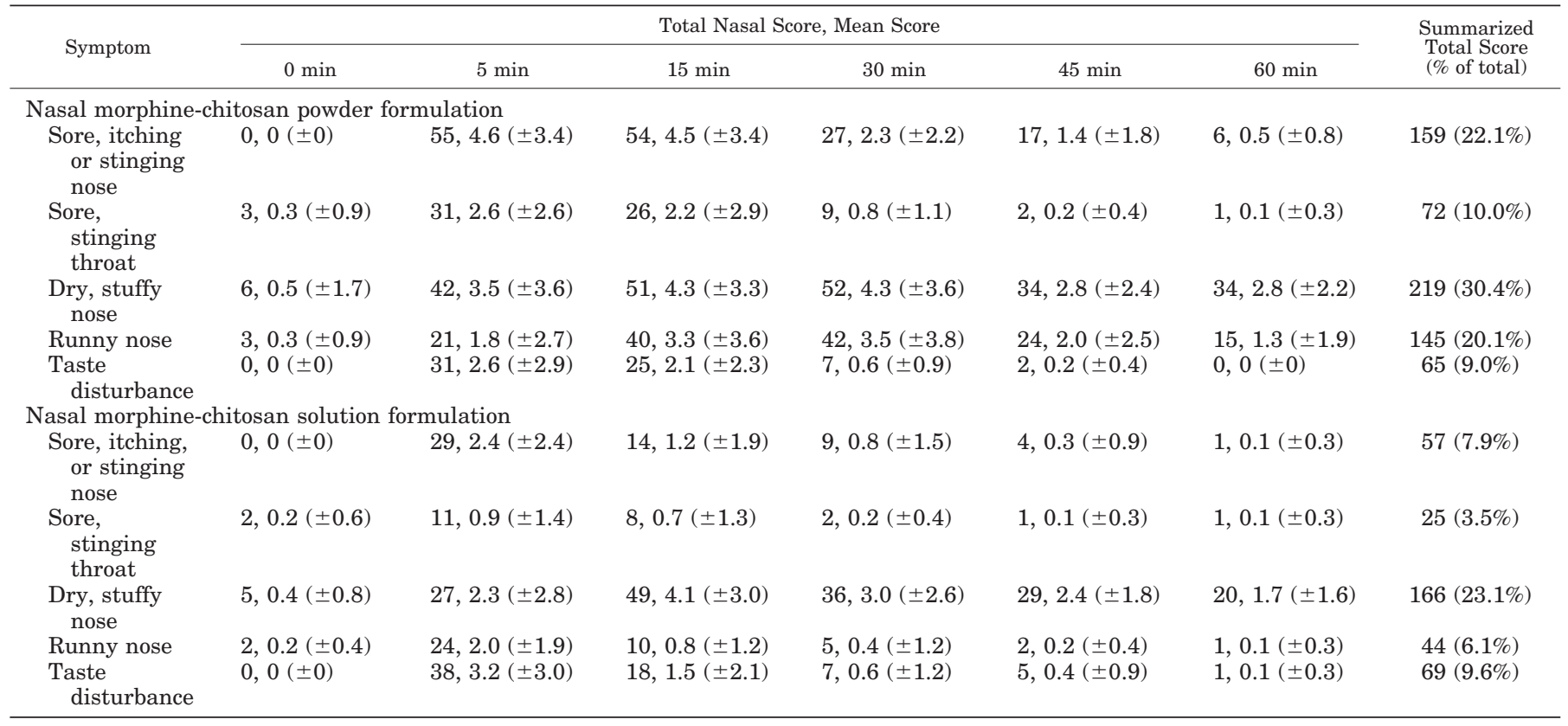

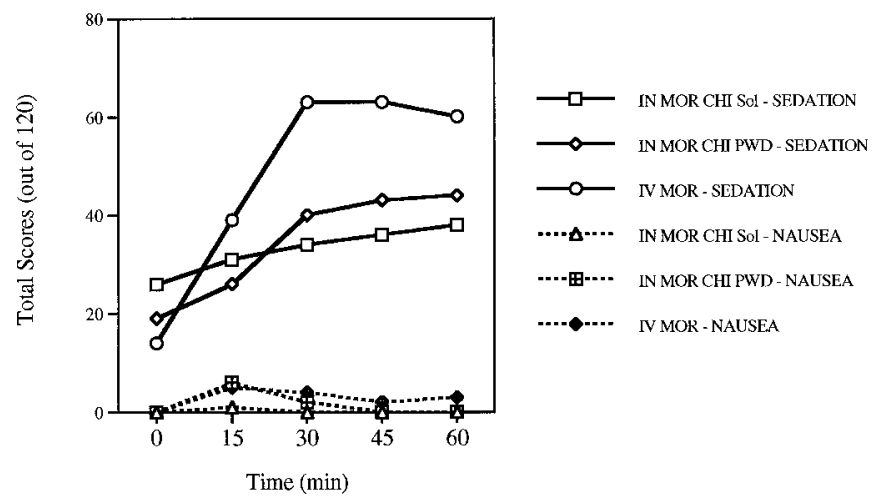

Fig. 3. Total central effects scores in human volunteers after nasal administration of a morphine solution formulation with chitosan, a powder formulation containing morphine and chitosan and an intravenous administration of morphine. IN MOR CHI PWD, intranasal morphinechitosan powder; IN MOR CHI Sol, intranasal morphine-chitosan solution; i.v. MOR, intravenous morphine.

(1995) (60\%) in a rat model and in rabbits by Chast et al. (1992) (86\%). This is most likely due to the use of anesthesia in the rat and rabbit models during administration of the nasal formulations. Anesthesia is known to give rise to a decrease in mucociliary clearance rate and has been shown to enhance the nasal absorption of drugs (Illum, 1996; Mayor and Illum, 1997). The sheep model used in these experiments only involved mild sedation for about 3 min during dosing and the model has been shown to be predictive of absorption in humans (Illum, 1996).

The administration of morphine in the chitosan solution formulation improved the nasal bioavailability in sheep about 3 times and also decreased the $T_{\max }$ to $14 \mathrm{~min}$. This improvement in nasal absorption was even more pronounced for the cross-linked chitosan powder formulation where the bioavailability reached $55 \%$ and the $T_{\max }$ was $7.5 \mathrm{~min}$. We believe that the improvement by chitosan of the nasal absorption of morphine is caused by two main mechanisms. First, chitosan is a mucoadhesive material that, because of the high density of the positive charges on the molecule, adheres strongly to negative sites on the nasal membrane such as sialic acid residues in mucin glycoproteins (Leung and Robinson, 1988; Ahuja et al., 1997; Illum, 1998a,b). This mucoadhesive property results in the nasally administered chitosan formulations having an increased clearance time (at least doubled), thereby promoting the nasal absorption of a drug (Soane et al., 1999, 2001). Second, it has been demonstrated that chitosan, when applied to confluent cell cultures, is able to transiently open the tight junctions between the cells (as verified by a decrease in transepithelial electrical resistance, increased transport of mannitol, and changes to the conformation of the junctional proteins) (Artursson et al., 1994; Borchard et al., 1996; Dodane et al., 1999). It likely that a similar effect on tight junctions can take place in vivo. This would explain the rapid rate of absorption seen in the present work.

The nasal formulation combining starch microspheres and the surfactant material LPC together with morphine as a freeze-dried powder resulted in the highest bioavailability of about $75 \%$ after nasal administration to sheep. The starch microspheres are known to be mucoadhesive and thereby provide a prolonged contact between the drug and the mucosa. Furthermore, after deposition on the surface of the nasal cavity the starch microspheres take up water from the mucous membrane, which may dehydrate the membrane and thereby "force open" tight junctions (Edman et al., 1992). It was shown by Illum et al. (2001) that a combination of the starch microspheres with LPC synergistically enhanced the absorption-promoting effect of both the microspheres and 

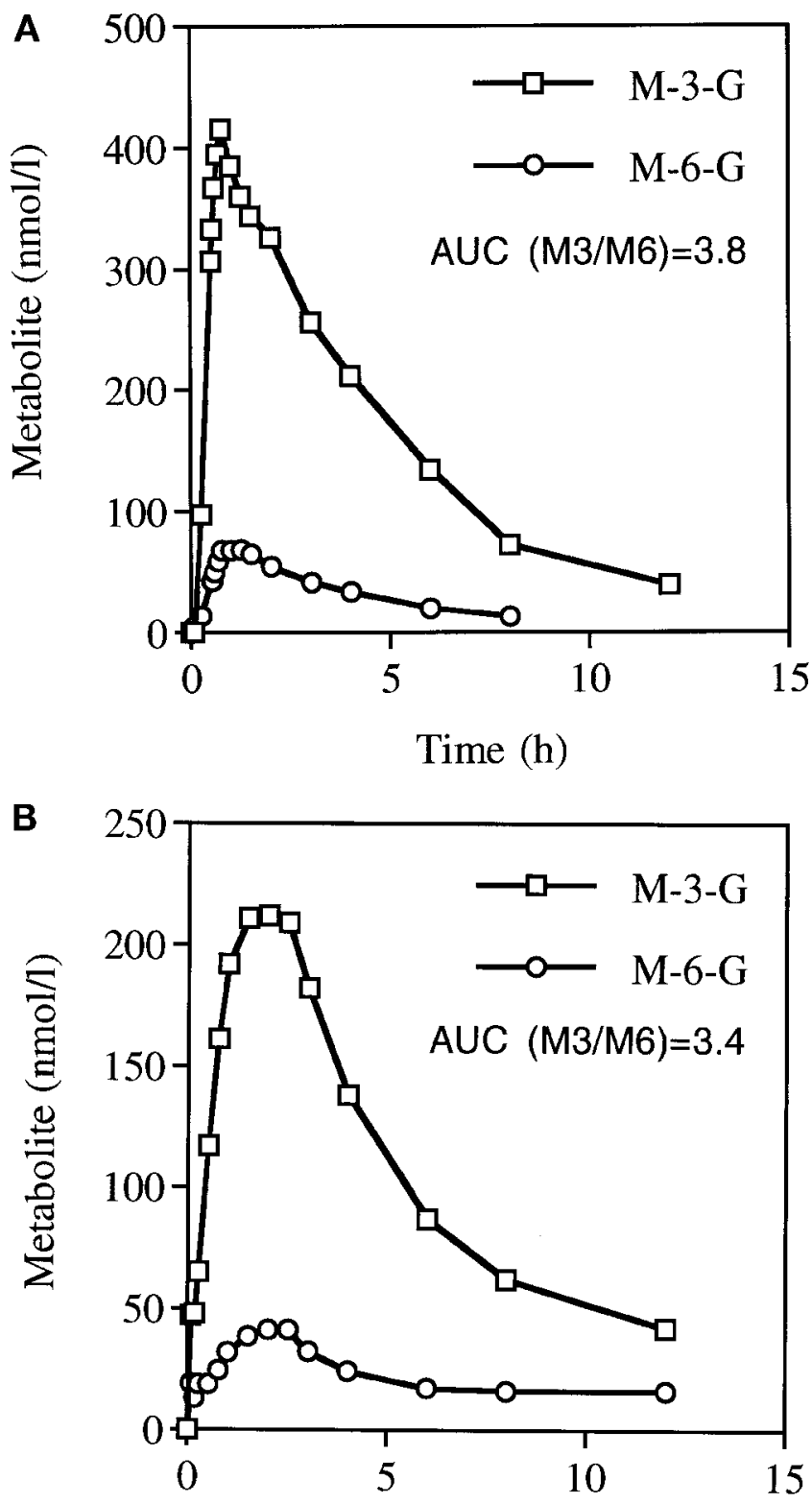

\section{Time (h)}

Fig. 4. Plasma concentration of morphine metabolites M-3-G and M-6-G after intravenous administration of morphine (A) and nasal administration of a morphine-chitosan solution formulation (B).

the surfactant absorption enhancer by 5 to 7 times. The LPC compound is believed to work by changing the physicochemical properties of the cell membrane lipid bilayer and possibly by opening tight junctions in the membrane (Marttin et al., 1995).

The starch microsphere LPC formulation was included in the sheep study to evaluate how high a bioavailability it would be possible to obtain. However, the LPC has been shown to exhibit some local toxicity on the nasal membrane and therefore, it was decided to select nasal morphine formulations based on chitosan for nasal pharmacokinetic studies in human volunteers. Chitosan has been shown in a range of toxicity studies to have a very safe toxicity profile and is in clinical development for a range of nasal products (Aspden et al., 1995, 1997a,b; Illum, 1998a,b).
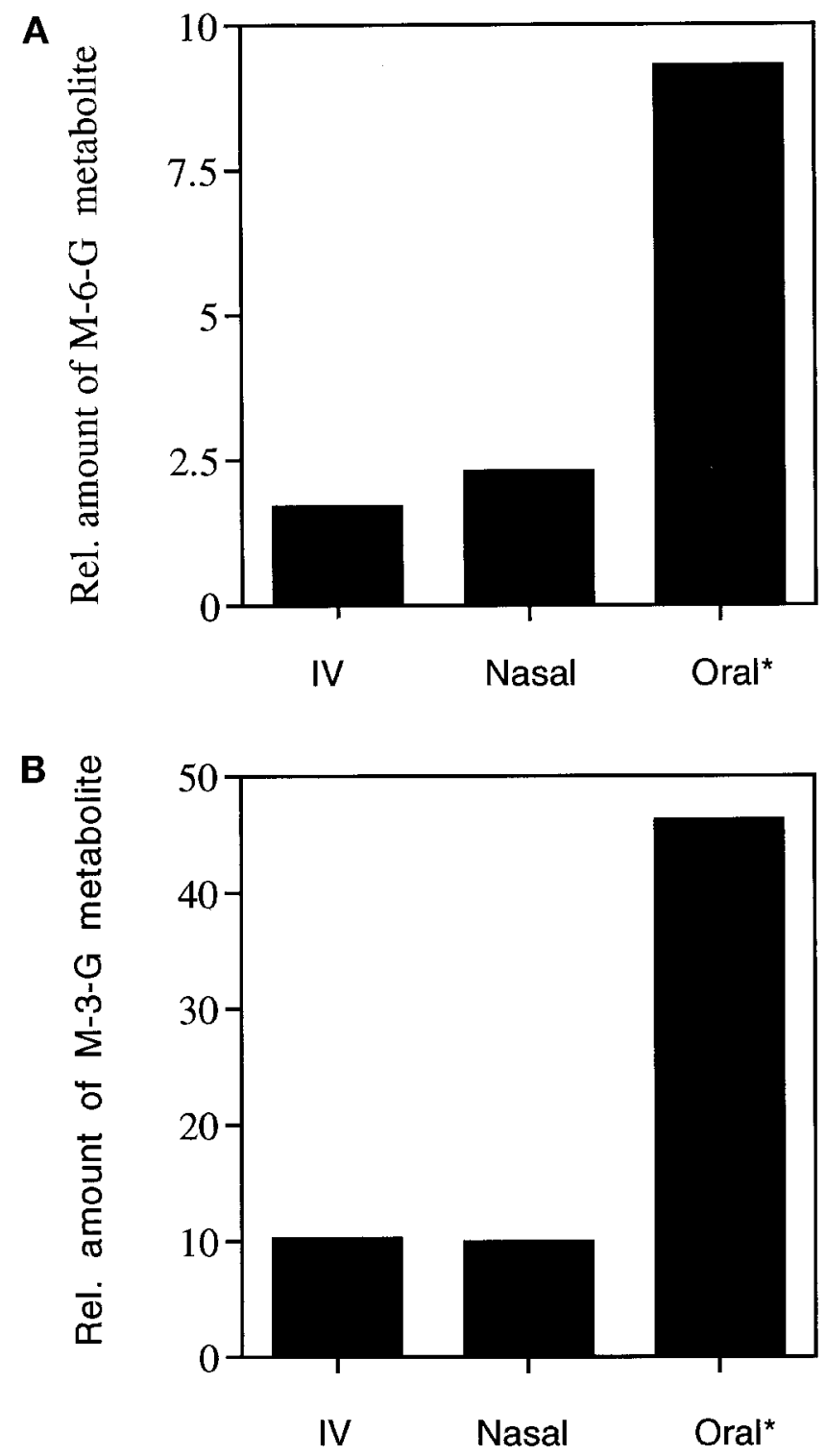

Fig. 5. Relative amount of morphine metabolites M-6-G (A) and M-3-G (B) after intravenous administration of morphine, nasal administration of a morphine-chitosan solution formulation, and oral administration of morphine. The oral data taken from Osborne et al. (1990).

Despite apparent improvements in F\% for most of the novel nasal morphine formulations compared with the morphine solution control, the lack of statistically significant findings between the control and the chitosan solution and chitosan microsphere-based morphine formulations could be attributed to high interanimal variability and the relatively small numbers of animals in each group. Based on the S.D. obtained for parameter F\%, group sizes of around 6 and 12 sheep would be required to demonstrate significant difference (at 70\% statistical power) between the control and the chitosan powder or chitosan solution formulation, respectively (GraphPad StatMate software). However, for purpose of screening nasal formulations, group sizes of more than six sheep are wasteful and impractical.

Studies in Volunteers. The human volunteers were given morphine doses of $10 \mathrm{mg}$ in three different formulations: a nasal solution formulation and a powder formulation both containing chitosan and morphine hydrochloride and an 
intravenous infusion of morphine sulfate over $30 \mathrm{~min}$, in a crossover design. The nasal solution and powder formulations resulted in substantially identical morphine plasma profiles with rapid and high peak plasma concentrations, which were similar in shape to the profile obtained for intravenous administration (Fig. 2). The $T_{\max }$ for the nasal powder formulation was slightly longer $(21 \mathrm{~min})$ than for the solution formulation (15 min), which would be expected for a mucoadhesive powder formulation. The reason why in humans the nasal powder formulation did not increase the absorption of the morphine to a higher degree than the chitosan solution formulation could partly be due to the similarities that exist between the physicochemical characteristics of the two chitosan formulations. The devices used for the solution and powder formulations in sheep and humans were different but both active in action. Different devices were necessary due to the different morphology of the nasal cavity of sheep and humans (Illum, 1996). However, it has been shown by our own group that both for solution and powder formulations the clearance times obtained in sheep and humans are very comparable when administered by such methods (Soane et al., 1999, 2001). Because the clearance of formulations from the nasal cavity is dependent upon the site of deposition, the comparability between the two species and the delivery device are evident. The increased clearance time of both the solution and powder chitosan formulations is reflected in the apparent prolonged half-life of the two nasal formulations compared with intravenous administration.

Of great interest in the present work are the low levels of the morphine metabolites M-3-G and M-6-G that were produced after nasal administration of the morphine formulations. The relative plasma levels of the two metabolites were very similar to the levels produced after intravenous injection and were much lower than those produced after oral administration of morphine (Figs. 4 and 5). It is the first time this similarity has been reported in the literature. This supports the view that the nasal morphine is absorbed directly from the nose into the systemic circulation and bypasses the gut wall and the liver and thereby first pass metabolism. This is further supported by the similarity between the ratio of the two metabolites after the intravenous injection and the nasal administration of the morphine formulation.

The tolerability of the formulations as experienced by the volunteers was generally good with the summarized total tolerance scores being less than $30 \%$ of the total scores obtainable. The morphine solution formulation containing chitosan was generally better tolerated than the powder formulation in that the combined scores for most categories were lower. This is not surprising considering that the morphine hydrochloride salt itself is somewhat irritating in the nasal cavity and that this sensation would be expected to be more pronounced when administered as a powder formulation. Recently, we have conducted a human tolerance study in 12 subjects that has examined the repeated administration of chitosan solution or placebo solution and chitosan powder or placebo powder systems without drug. Both formulations were well tolerated and provided very low tolerance scores (data not shown).

The main central effect recorded in the volunteer questionnaire was that of sedation. The sedation was most pronounced after the intravenous administration of morphine but still prominent for all three administrations even at 60 min postadministration. However, of interest is the fact that the scores among the volunteers were higher at the earliest time point after nasal administration compared with intravenous administration. This suggests that after nasal administration morphine may be able to reach the central nervous system more rapidly than after intravenous administration, where the morphine has to pass the blood-brain barrier (although it has to be born in mind that the intravenous injection was given as an infusion over $30 \mathrm{~min}$ ). Very few studies have been carried out on the transport of morphine from nose to brain. It has been shown that drugs such as cocaine (at the lower end of the lipophilicity scale) have a higher cerebrospinal fluid and olfactory bulb concentration after nasal administration than that obtained after parenteral administration, especially during the first few minutes after application (Chow et al., 1999). The most likely pathways followed by such drugs will be transcellular and/or paracellular routes across the olfactory epithelium (Illum, 2000). However, such a possible nose-to-brain transport warrants further investigation.

The nasal solution formulation containing morphine and chitosan has recently been tested in a pilot study in cancer patients for the treatment of breakthrough pain (Wilcock et al., 2001). Fourteen cancer patients were treated with various doses of morphine (5-80 mg) in 20 episodes of breakthrough pain. These patients were normally treated with oral morphine for episodes of breakthrough pain in addition to baseline therapy with strong opioids. The efficacy and tolerability of the nasal formulation were determined using pain scores for up to $4 \mathrm{~h}$ after dose administration. It was found that the onset of pain relief was rapid with the mean pain intensity scores decreasing from between "moderate-to-severe" pain to between "slight-to-moderate" pain within 5 min. The nasal formulations were well tolerated by the patients and the satisfaction with nasal administration of morphine was high.

\section{Conclusion}

It can be concluded from these studies that it is possible with a nasal morphine formulation containing chitosan to obtain a rapid and therapeutically relevant peak plasma level of morphine. The plasma profiles after nasal administration were similar to those obtained after intravenous administration of morphine and a bioavailability of about $60 \%$ can be obtained. The pharmacokinetic data from the sheep and human studies was subjected to statistical analysis. Pilot studies in cancer patients have shown the efficacy of the nasal morphine formulation as a means of improving the treatment of breakthrough pain. The nasal morphine formulation containing chitosan has been shown to be well tolerated and well accepted by both volunteer subjects and cancer patients.

\section{References}

Ahuja A, Khar RK, and Ali J (1997) Mucoadhesive drug delivery systems. Drug Del Indust Pharm 23:489-515.

Artursson P, Lindmark T, Davis SS, and Illum L (1994) Effect of chitosan on the permeability of monolayers of intestinal epithelial cells (Caco-2). Pharm Res 11: 1358-1361.

Aspden TJ, Adler J, Davis SS, Skaugrud O, and Illum L (1995) Chitosan as a nasal delivery system: evaluation of the effect of chitosan on mucociliary clearance in the frog palate model. Int $J$ Pharm 122:69-78.

Aspden TJ, Illum L, and Skaugrud O (1997b) The effect of chronic nasal application of chitosan solutions on cilia beat frequency in guinea pigs. Int J Pharm 153:137146. 
Aspden TJ, Mason JDT, Jones NS, Lowe J, Skaugrud O, and Illum L (1997a) Chitosan as a nasal delivery system: the effect of chitosan solutions on the in vitro and in vivo mucociliary transport rates in human turbinates and volunteers. J Pharm Sci 86:509-513.

Behl, C (2000) Experiences and the Potential of Nasal Drug Delivery, in Presentation to a Management Forum Meeting on Nasal Drug Delivery; 2000 March 23-24; London. Management Forum.

Borchard G, Luessen HL, deBoer AG, Verhoef JC, Lehr CM, and Junginger HE (1996) The potential of mucoadhesive polymers in enhancing intestinal peptide drug absorption. III effects of chitosan-glutamate and carbomer on epithelial tight junctions in vitro. $J$ Control Rel 39:131-138.

Bourget P, Lesne-Hulin A, and Quinquis-Desmaris V (1995) Study of the bioequivalence of two controlled-release formulations of morphine. Int $J$ Clin Pharmacol Ther 33:588-594.

Chast F, Bardin C, Dume L, and Sauvageon-Martre H (1992) Les voies d'administration non conventionelles da la morphine. J Pharm Clin 11:257-261.

Chow H-HS, Chen Z, and Natsuura GT (1999) Direct transport of cocaine from the nasal cavity to the brain following intranasal cocaine administration in rats. J Pharm Sci 88:754-758.

Cleary JF (1997) Pharmacokinetic and pharmacodynamic issues in the treatment of breakthrough pain. Semin Oncol 24:S16-13-S16-19.

Dodane V, Khan MA, and Merwin JR (1999) Effect of chitosan on epithelial permeability and structure. Int $J$ Pharm 182:21-32.

Edman P, Bjork E, and Ryden L (1992) Microspheres as a nasal delivery system for peptide drugs. J Control Rel 21:165-172.

Faura CC, Moore RA, Horga JF, Hand CW, and McQuay HJ (1996) Morphine and morphine-6-glucuronide plasma concentrations and effect in cancer Pain. J Pain Symptom Manage 11:95-102.

Foley KM (1995) Pain relief into practice: rhetoric without reform. J Clin Oncol 13:2149-2151.

Illum L (1996) Animal models for nasal delivery. J Drug Target 3:717-724.

Illum L (1998a) Bioadhesive formulations for nasal peptide delivery, in Bioadhesion in Drug Delivery (Frokjaer S, Christrup L, and Krogsgaard-Larsen P eds) pp 157-170, Munksgaard, Copenhagen.

Illum L (1998b) Chitosan and its use as a pharmaceutical excipient. Pharm Res 15:161-170

Illum L (2000) Transport of drugs from the nasal cavity to the central nervous system. Eur J Pharm Sci 11:1-18.

Illum L, Davis SS, Pawula M, Fisher AN, Barrett DA, Farraj NF, and Shaw PN (1996) Nasal administration of morphine-6-glucuronide in sheep - a pharmacokinetic study. Biopharm Drug Dispos 17:717-724.

Illum L, Farraj NF, and Davis SS (1994) Chitosan as a novel nasal delivery system for peptide drugs. Pharm Res 11:1186-1189.

Illum L, Fisher AN, Jabbal-Gill I, and Davis SS (2001) The effect of bioadhesive starch microspheres on the absorption enhancing effect of enhancing agents administered nasally. Int $J$ Pharm 222:109-119.

Illum L, Watts P, Fisher AN, Jabbal-Gill I, and Davis SS (2000) Novel chitosan based delivery systems for nasal administration of a LHRH-analogue. STP Pharma 10:89-94.

Kondo T, Nishimura K, Irie T, and Uekama K (1995) Cyclodextrin derivatives that modify nasal absorption of morphine and its entry into cerebrospinal fluid in the rat. Pharm Sci 1:163-166.
Leung SHS and Robinson JR (1988) The contribution of anionic polymer structural features to mucoadhesion. J Control Rel 5:223-231.

Marttin E, Verhoef JC, Romeijn SG, Zwart P, and Merkus FWHM (1995) Effects of absorption enhancers on rat nasal epithelium in vivo: release of marker compounds in the nasal cavity. Pharm Res 12:1151-1157.

Mayor SH and Illum L (1997) An investigation of the effect of anaesthetics on the nasal absorption of insulin in rats. Int J Pharm 149:123-129.

Murphey LJ, Olsen GD, and Konkol RJ (1993) Quantitation of benzoylnorecgonine and other cocaine metabolites in meconium by high performance liquid chromatography. J Chromatogr 613:330-335.

Osborne R, Joel S, Trew D, and Slevin M (1990) Morphine and metabolite behaviour after different routes of morphine administration: demonstration of the importance of the active metabolite morphine-6-glucuronide. Clin Pharmacol Ther 47: $12-19$.

Roon KI, Soons PA, Uitendaal MP, de Beukelaar F, and Ferrari MD (1999) Pharmacokinetic profile of alniditan nasal spray during and outside migraine attacks. Br J Clin Pharmacol 47:285-290.

Shyu WC, Pittman KA, Robinson D, and Barbhaiya RH (1993) Multiple-dose phase I study of intranasal butorphanol. Clin Pharmacol Ther 54:34-41.

Soane RJ, Frier M, Perkins AC, Jones NS, Davis SS, and Illum L (1999) Evaluation of the clearance characteristics of bioadhesive systems in humans. Int $J$ Pharm 178:55-65.

Soane RJ, Hinchcliffe M, Davis SS, and Illum L (2001) Clearance characteristics of chitosan based formulations in the sheep nasal cavity. Int $J$ Pharm 217:183-191.

Svensson JO, Rane A, and Sjoqvist F (1982) Determination of morphine, morphine3glucuronide and (tentatively) morphine-6-glucuronide in plasma and urine using ion-pair high-performance liquid chromatography. J Chromatogr 230:427-432.

Takala A, Kaasalainen V, Seppala T, Kalso E, and Olkkola KT (1997) Pharmacokinetic comparison of intravenous and intranasal administration of oxycodone. Acta Anaesthesiol Scand 41:309-312.

Todd RD, Muldoon SM, and Watson RL (1982) Determination of morphine in cerebrospinal fluid and plasma by high-performance liquid chromatography with electrochemical detection. J Chromatogr 232:101-110.

Twycross RG (1994) Opioids, in Textbook of Pain (Wall PD and Melzack R eds) pp 943-962, Churchill Livingstone, London.

Westerling D, Persson C, and Hoglund P (1995) Plasma concentrations of morphine, morphine-3-glucuronide, and morphine-6-glucuronide after intravenous and oral administration to healthy volunteers: relationship to nonanalgesic actions. Ther Drug Monogr 17:287-301.

Wilcock A, Pavis H, Edgecombe J, Carr D, Manderson C, and Church A (2001) Nasal morphine for the treatment of breakthrough pain in cancer patients. J Pain Symptom Manage, in press.

World Health Organization (1986). Cancer Pain Relief Program. World Health Organization, Geneva, Switzerland.

Address correspondence to: Dr. Lisbeth Illum, West Pharmaceutical Services, Drug Delivery and Clinical Research Center Ltd., Albert Einstein Center, Nottingham Science and Technology Park, Nottingham NG7 2TN, UK. E-mail: lisbeth_illum@westpharma.com 